\section{Happy to be extracted}

Sir, this permanent maxillary first molar was removed from a 67-year-old patient, without complication (Fig. 1). This smiling tooth appeared to be very happy to be leaving the patient pain-free. Seeing this tooth really bought some festive cheer to our OMFS department and we share this whimsical photograph in the hope that it will do the same for $B D J$ readers. We wonder if other facial expressions have been displayed on extracted teeth!

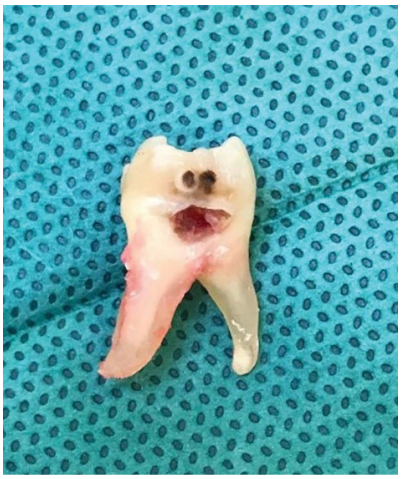

Fig. 1 A very happy molar

N. Dhillon, A. Cameron, A. N. Beech, Gloucester, UK

https://doi.org/10.1038/s41415-022-3836-3

\section{Occupational health}

\section{Refine Achenbach searches}

Sir, the recent letter by Y. A. Picón-Jaimes et al. ${ }^{1}$ has complemented our earlier case report on Achenbach's syndrome, ${ }^{2}$ by drawing attention to a publication that we had missed and which illustrated a single case of Achenbach's syndrome that had an association with recurrent subconjunctival haemorrhages. ${ }^{3}$

The authors had also previously written to the Journal of the Royal Society of Medicine Open (JRSM Open) with the same notification, ${ }^{4}$ in response to our original, more detailed publication on the possible association between Achenbach's syndrome and joint hypermobility. ${ }^{5}$

In that JRSM Open letter, ${ }^{4}$ HigueraCetina et al. had also cited a report that illustrated a familial association between three related women, which had suggested a possible genetic cause for their finger bleeds. ${ }^{6}$ As such, they then felt this hypothesis had been reinforced by our report of the two female sibling Achenbach cases who had also co-presented with joint hypermobility. ${ }^{5}$ However, this is not surprising bearing in mind that many regard joint hypermobility syndrome as being synonymous with Ehlers-Danlos Type III syndrome, which is inherited through an autosomal dominant pathway, even if the genes responsible have yet to be identified.?

As such, in light of the above two additional associations of subconjunctival haemorrhages and a familial trait to screen for in patients who report with paroxysmal, subcutaneous finger bleeds, we still believe the main focus of attention should be on searching for more cases that may also present with joint hypermobility, in order to further substantiate the existence of an association with Achenbach's syndrome, as a means thereafter if found, of encouraging the initiation of subsequent research into the possible pathophysiological mechanisms of its causation.

\section{R. A. C. Chate, C. Chate, Colchester, UK}

\section{References}

1. Picón-Jaimes $Y$ A, Orozco-Chinome J E, LozadaMartinez I D, Moscote-Salazar L R. Achenbach's other lesions. Br Dent J 2021; 231: 534-535.

2. Chate $R$, Chate $C$. Achenbach's syndrome. Br Dent J 2021; 231: 147

3. Young B, Okera S. Recurrent subconjunctival haemorrhage in Achenbach's Syndrome. Clin Exp Ophthalmol 2018; 46: 965-966.

4. Higuera-Cetina C I, Montaña-Gómez L M, Picón-Jaimes Y A, Orozco-Chinome JE, LozadaMartinez I, Moscote-Salazar L R. Achenbach's Syndrome: a rare condition. JRSM Open 2021; doi: 10.1177/20542704211047121.

5. Chate R A C. Achenbach's hand and digital paroxysmal haematomas: a possible association with joint hypermobility syndrome in two sibling cases. JRSM Open 2021; doi: 10.1177/20542704211023162.

6. Helm R H. Achenbach syndrome: a report of three familial cases. J Hand Surg Eur Vol 2021; doi: 10.1177/17531934211024567.

7. Malfait $F$, Francomano C, Byers P et al. The 2017 International Classification of the Ehlers-Danlos Syndromes. Am J Med Genet C Semin Med Genet 2017; 175: 8-26.

https://doi.org/10.1038/s41415-022-3837-2

\section{Dental sleep medicine}

\section{Pertinent points}

Sir, firstly, congratulations to Dr Leigh et al. ${ }^{1}$ on their paper which I thought raised several very pertinent points in the developing field of dental sleep medicine. I quite agree that the lack of undergraduate education makes it incumbent on a clinician to seek suitable training prior to entering the field of sleep dentistry.

I would like to make your readers aware of the British Society of Dental Sleep
Medicine (BSDSM) which has been in existence for several years with the aim of providing independent advice and non-commercial courses for dentists. We provide regular introductory courses and have a Facebook group for members to network and discuss cases. We are a nonprofit organisation and also have a website listing members who offer sleep medicine services to the general public, for which all dentists have attended suitable training courses and maintained postgraduate activity. We have also produced a care pathway and management protocol which has been approved by several leading indemnifiers.

Additionally, we recently affiliated with the British Sleep Society (BSS) as both bodies recognised the need for an MDT approach particularly in light of the recent NICE guidelines. Membership is open and available on our website.

J. Bowker, Secretary BSDSM, Liverpool, UK

\section{Reference}

1. Leigh S, Faigenblum $M$, Fine $P$, Blizard $R$, Leung $A$. General dental practitioners' knowledge and opinions of snoring and sleep-related breathing disorders. $\mathrm{Br}$ Dent J 2021; 231: 569-574. https://doi.org/10.1038/s41415-022-3838-1

\section{Oral health}

\section{Vaping: should we be worried?}

Sir, there has been a surge in popularity as of late in 'disposable' vape bars. These are sold in convenience stores and come in a variety of flavours. They can also be purchased online (in some cases, without age verification). The majority of these products contain $20 \mathrm{mg}$ of nicotine salt, with a total of 600 puffs - equivalent to 45 cigarettes. The addition of an integrated e-liquid and power supply means they can be discarded following usage.

E-cigarette exposure can increase the risk for deteriorating periodontal health as well as changes to the oral microbiome. Components of e-cigarette vapour also contain known cytotoxic, genotoxic and carcinogenic properties. ${ }^{1}$ Furthermore, disposable systems can deliver nicotine at a higher rate per puff to the user than traditional cigarettes, which may lead to an increased dependency. ${ }^{2}$

Vape bars are becoming increasingly popular amongst teenagers who are unlikely to be using them for their intended 